\title{
Editorial
}

\section{Postoperative anaesthesia care}

In an attempt to reduce perioperative cardiac morbidity, intense study and research has been centred, practically exclusively, on the anaesthetic management during the preoperative and intraoperative periods. The postoperative period has been virtually ignored. However, recent data strongly suggest that we should be directing the same intense interest to management of the immediate postoperative period. In 1990 Mangano et al.' reported on their study of 474 male patients with either known coronary artery disease (CAD) (243) or at high risk for it (231) undergoing elective non-cardiac surgery. In this study $41 \%$ of patients had postoperative myocardial ischaemia compared with only $20 \%$ preoperatively and $25 \%$ intraoperatively. Of more importance, these ischaemic episodes were silent in $97 \%$ of patients. Furthermore, they found a threefold increase in the likelihood of having an adverse cardiac outcome associated with the occurrence of postoperative ischaemia, similar to that shown with patients having cardiac surgery.

These findings are not so surprising when we remember that the postoperative period is associated with major changes in adrenergic activity, plasma catecholamine concentrations, body temperature, pulmonary function, fluid balance and perception of pain. ${ }^{2}$ Fluctuations of these factors can lead to an imbalance between the relationship of myocardial oxygen supply and demand, thus promoting the likelihood of the development of myocardial ischaemia. It may be that by blunting the activation of the sympathetic nervous system in the postoperative period, thus preventing the resultant endocrine, metabolic and haemodynamic changes, this imbalance might be shifted away from ischaemia. Since the introduction of regional anaesthesia techniques, it has been suggested that their use may reduce postoperative morbidity and even mortality in high risk surgical patients. Whether or not this is true has been the subject of much debate, research and controversy!

It is well established that patients undergoing major

From the Department of Anaesthesia, Royal Victoria Hospital, 687 Pine Avenue Wcst, Montreal, Quebec H3A IAI vascular surgery are at increased risk for postoperative cardiac complications, ${ }^{3}$ presumably due to the high prevalence of severe coronary artery disease in this population. ${ }^{4}$ In this issue of the journal, Garnett et al. ${ }^{5}$ report their findings comparing the use of combined general and epidural anaesthesia with postoperative epidural analgesia, to general anaesthesia and postoperative intravenous analgesia, on the incidence of perioperative myocardial ischaemia in patients undergoing elective aortic reconstructive surgery. The goal of this study was to determine if a specific anaesthetic technique and postoperative pain management could effect the incjdence of perioperative myocardial ischaemia. The authors studied 99 patients undergoing elective aortic reconstructive surgery. Patients were randomly assigned to one of two group, to receive either general and epidural anaesthesia with postoperative epidural analgesia (EPI), or general anaesthesia with postoperative intravenous analgesia (GA). Patients in the epidural group $(n=48)$ received a loading dose of $10-15 \mathrm{ml}$ carbonated lidocaine $2 \%$ and $2 \mathrm{mg} \cdot \mathrm{ml}^{-1}$ meperidine followed by a continuous infusion of 5-12 $\mathrm{ml} \mathrm{hr}^{-1}$ lidocaine/meperidine via the epidural catheter. All patients in both groups had a standardized anaesthetic induction of 2-3 mg midazolam, 20-30 $\mathrm{g} \mathrm{kg}^{-1}$ fentanyl and $0.15-0.2 \mathrm{mg} \cdot \mathrm{kg}^{-1}$ pancuronium. Additional doses of fentanyl $\left(3-6 \mu \mathrm{g} \cdot \mathrm{kg}^{-1}\right)$ were administered in the general anaesthesia only group. Haemodynamic variables were controlled to remain within $\pm 20 \%$ of baseline values and stroke volume $>1 \mathrm{ml} \cdot \mathrm{kg}^{-1}$. All patients had a three lead Holter monitor (leads II, $V_{2}$ and $V_{5}$ ) attached the day before surgery and worn continuously for $96 \mathrm{hr}$. Outcome factors were mortality and cardiac morbidity defined as myocardial infarction, angina, congestive heart failure or serious arrhythmias (ventricular tachycardia or fibrillation, atrial fibrillation or flutter).

In contrast to other studies the authors subjectively sought myocardial ischaemia with the use of a continuous Holter monitor for $40-44 \mathrm{hr}$ postoperatively. They found no difference in the incidence of perioperative myocardial ischaemia between the two groups $(51 \%$ in the GA group and 58\% in the EPI group). However, ischaemia was not a predictor of myocardial infarction. 
Only six (11\%) of the 54 patients who had at least one ischaemic episode, had a myocardial infarction. Also, of the 44 patients who had no perioperative ischaemic event, two (4.4\%) suffered myocardial infarction. Of note, all six of the myocardial infarctions that occurred in the monitored period were associated with tachycardia. Furthermore, an increase in heart rate of $50-60 \%$ over the preoperative heart rate produced a $50 \%$ risk of ischaemia. Their most important finding was the burst of ischaemic events -60 in nine patients in the EPI group - after the epidural infusion was discontinued. This time was associated with an increase in both heart rate and blood pressure, which may reflect the return of sympathetic tone and increased pain perception. More importantly, removal of these catheters is normally at a time when most cardiac monitors are discontinued, prior to transfer from the ICU and, therefore, these ischaemic episodes would normally go undetected. This suggests the need for early analgesia to reduce the severity of the return of pain and its associated stresses or the ability to continue the use of epidural analgesia once the patient has been discharged from the ICU.

The authors also looked for specific events in the perioperative period that were associated with myocardial ischaemia. Perhaps the most significant and worrisome finding in this paper is their observed high risk postoperative periods. These risk periods included transfer to and from the ICU (not unexpected) but also during physiotherapy and bed baths! As pertinently commented by the authors, two procedures that appear to do more harm than good! Clearly, detection of myocardial ischaemia is an important goal in its prevention. How can it best be detected in the postoperative period? As the majority of ischaemic episodes have been shown to be silent,' the patients are unreliable detectors. In addition, detection of ischaemic episodes by the medical staff from routine ECG monitoring is notoriously poor. It may be that an acute awareness of both these expected and unexpected high risk periods as described in the paper in this journal is all that we can have at the moment.

Why did these results not agree with those of previous studies, which have shown decreased morbidity and mortality in patients receiving an epidural anaesthetic technique? Many of the previous studies have not been restricted to one type of surgery as was the case in this study. In their landmark study in 1987, Yeager at al. ${ }^{6}$ found a reduction in postoperative mortality, cardiovascular and respiratory complications and major infection in patients receiving epidural anaesthesia and postoperative epidural analgesia compared with patients receiving general anaesthesia and prn opioid analgesia postoperatively. However, when one reviews patient management in their study there are several factors that may have inadvertently contributed to their results and the inability of subsequent studies to confirm their findings.

Firstly, these authors studied a small number of patients undergoing a wide range of procedures. Also general anaesthetic techniques and the use of invasive monitoring were poorly controlled perioperatively. It has been well documented that the use of invasive monitoring in high risk patients may reduce perioperative cardiac complications considerably. ${ }^{7}$ Finally, postoperative pain management protocols were not comparable. Patients in the epidural group were followed daily by an acute pain service team in contrast to receiving $i v$ opioids prn administered by nurses. In fact, it may be exactly this difference in postoperative care that contributed to their findings. Subsequent studies have continually emphasised the importance of postoperative pain management as an important factor in postoperative morbidity and mortality. ${ }^{8.9}$ Baron et al ${ }^{8}$ in a study of 173 patients scheduled for abdominal aortic reconstruction surgery concluded that thoracic epidural anaesthesia in combination with light general anaesthesia is not preferable to general anaesthesia in high-risk surgical patients. However, in this study, the postoperative protocol for analgesia was not dictated by the study protocol. Therefore, three different analgesic techniques were distributed equally in both the epidural and the general anaesthesia groups. The authors suggest that postoperative epidural analgesia, rather than intraoperative epidural anaesthesia, played a major role in their incidence of postoperative cardiac complications.

It now appears that postoperative pain management per se plays a more important role in postoperative events than the type of anaesthesia used intraoperatively, so long as intraoperative haemodynamic stability is well maintained. This lack of effect of choice of anaesthetic technique on operative outcome, if haemodynamic variables are well controlled, has been demonstrated in patients undergoing cardiac surgery. ${ }^{10}$ Is it so surprising to have a similar result in patients with cardiac disease having non cardiac surgery?

What additional information is gained from the paper in this journal by Garnett et al. ${ }^{5}$ Firstly, compared with carefully conducted general anaesthesia, a regional anaesthetic technique appears to bestow no additional benefit on patient outcome. Obviously, there are other advantages to regional anaesthesia outside the possibility of reducing cardiac morbidity and mortality which need to be considered - not withstanding patients' choice. Christopherson et al." found a higher rate of graft occlusion among patients undergoing elective vascular reconstruction of the lower extremities, who received general anaesthesia compared with those who 
received a regional technique. They concluded that, by decreasing or preventing the neural-hormonal-metabolic stress response, the use of a regional anaesthetic technique shifted the hypercoagulative and fibrinolytic processes associated with this stress response back towards normal. Secondly, it is becoming obvious that we need to set clinical standards for intraoperative haemodynamic stability and for the management of postoperative pain. Finally, and of paramount importance, good postoperative pain control is an important factor in decreasing postoperative cardiac morbidity and mortality.

At last, the question of the effect of regional vs general anaesthesia on patient outcome can be laid to rest. It is becoming clear that the real issue for debate is not whether regional vs general anaesthesia is better but, rather, what is the optimal, overall management of the patient during the perioperative period. Therefore, anaesthetic care can no longer stop at the door of the operating room. To this aim increased attention and resources must be focused on the postoperative period.

\section{Les soins postanesthésiques}

En anesthésie, les études et travaux de recherche entrepris dans le but de diminuer la morbidité cardiaque périopératoire se sont presque entièrement limités à la prise en charge des périodes pré et peropératoire. En 1990, Mangano et al. ' ont publié le compte rendu d'une étude portant sur 474 coronariens connus (243) du sexe masculin ou à haut risque de maladie coronaire (231). Au cours de cette étude, $42 \%$ des patients ont présenté une ischémie myocardique en postopératoire comparativement à seulement $20 \%$ en préopératoire et $25 \%$ en peropératoire. Et ce qui est encore plus important, ces épisodes étaient silencieux dans $97 \%$ des cas. En outre, la probabilité d'une évolution cardiaque défavorable associée à l'apparition d'ischémie postopératoire triplait, c-à-d. qu'elle rejoignait celle des opérés sur le coeur.

Ces constatations n'ont rien de surprenant si on se rappelle que la période postopératoire est associée à des changements majeurs de l'activité adrénergique, des concentrations des catécholamines plasmatiques, de la température corporelle, de la fonction pulmonaire, de l'équilibre hydrique et de la perception de la douleur. ${ }^{2}$
La fluctuation de ces facteur peut provoquer au myocarde un déséquilibre entre l'apport et la demande en oxygène, et conséquemment de l'ischémie myocardique. Il se peut qu'en émoussant l'activation du système sympathique à la période postopératoire et en prévenant ainsi les changements endocriniens, métaboliques et hémodynamiques, ce déséquilibre soit déplacé hors du cycle de l'ischémie. L'introduction des techniques régionales nous a permis de penser qu'elles pourraient, chez les patients à haut risque, réduire la morbidité et même la mortalité postopératoires. Cette question n'a cessé de susciter des polémiques et d'inspirer des projets de recherches.

Il est bien établi que les opérés pour une chirurgie vasculaire majeure présentent un risque élevé de complications cardiaques postopératoires, ${ }^{3}$ vraisemblablement à cause de la prévalence élevée d'insuffisance coronarienne dans cette population. ${ }^{4}$ Dans ce numéro du Journal, Garnett $e t$ al. font le compte rendu d'une étude portant sur les effets comparés de l'anesthésie générale combinée à l'épidurale pour l'analgésie postopératoire à ceux de l'anesthésie générale combinée à l'analgésie postopératoire intraveineuse, sur l'incidence de l'ischémie myocardique périopératoire chez des patients soumis à une reconstruction chirurgicale programmée de l'aorte. Cette étude visait à déterminer si une technique anesthésique et une gestion analgésique postopératoire spécifiques pouvaient altérer l'incidence de l'ischémie myocardique postopératoire. Les auteurs ont fait porter leur étude sur 99 opérés pour une reconstruction chirurgicale programmée de l'aorte. Ils étaient assignés aléatoirement à un de deux groupes, dont un (EPI) recevait une anesthésie générale avec une analgésie épidurale pour l'analgésie postopératoire, et l'autre, une anesthésie générale avec une analgésie postopératoire intraveineuse (AG). Les patients du groupe épidural $(n=$ 48) recevaient une dose de charge de $10-15 \mathrm{ml}$ de lidocaïne carbonatée à $2 \%$ et $2 \mathrm{mg} \cdot \mathrm{ml}^{-1}$ de mépéridine suivie d'une perfusion continue par cathéter épidural de 5-12 $\mathrm{ml} \cdot \mathrm{hr}^{-1}$ de lidocaïne/mépéridine. Pour tous les patients des deux groupes, l'induction était standardisée et comprenait du midazolam 2-3 ml, du fentanyl 20-30 $\mu \mathrm{g} \cdot \mathrm{kg}^{-1}$ et du pancuronium $0,15-0,2 \mathrm{mg} \cdot \mathrm{kg}^{-1}$. Des doses supplémentaires de fentanyl $\left(3-6 \mu \mathrm{g} \cdot \mathrm{kg}^{-1}\right)$ étaient administrées au groupe AG. Les paramètres hémodynamiques étaient contrôlés de façon à maintenir les valeurs à $\pm 20 \%$ des valeurs initiales et un volume d'ćjection plus élevé que $1 \mathrm{ml} \cdot \mathrm{kg}^{-1}$. Tous les patients étaient reliés à un moniteur de Holter à trois dérivations (II, $V_{2}$ et $V_{5}$ ) installé la veille de la chirurgie et le conservaient pour $96 \mathrm{~h}$. On mesurait les résultats en fonction de la mortalité et de la morbidité cardiaques telles que définies plus spécifiquement par l'infarctus du 
myocarde, l'angine, l'insuffisance cardiaque congestive ou les arythmie graves (tachycardie ou fibrillation ventriculaire, fibrillation ou flutter auriculaire).

Contrairement aux autres études, les auteurs ont recherché l'ischémie myocardique avec un moniteur de Holter en continu pour $40-44 \mathrm{~h}$ en postopératoire. L'incidence de l'ischémie myocardique periopératoire n'a pas été différente entre les deux groupes $(51 \%$ dans le groupe $A G$ et $58 \%$ dans le groupe EPI). Cependant, l'ischémie n'avait aucune valeur prédictive pour l'infarctus du myocarde. Seulement six $(11 \%)$ des 54 patients qui avaient souffert d'au moins un épisode ischémique ont subi un infarctus du myocarde. Il est important de noter que l'infarctus du myocarde survenant pendant la période monitorée était associé à de la tachycardie. De plus, toute augmentation de la fréquence cardiaque de 50-60\% au-dessus de la valeur préopératoire initiale augmentait de $50 \%$ le risque d'ischémie. Leur constatation la plus importante est l'explosion des accès ischémiques après l'arrêt de la perfusion épidurale - $60 \mathrm{chez}$ neuf patients. Cette période était associée à une augmentation simultanée de la fréquence cardiaque et de la pression artérielle, ce qui peut être le reflet de la reprise du tonus sympathique et de la perception douloureuse. Ce qui est encore plus important, c'est que le retrait des cathéters se fait normalement à un moment où la plupart des moniteurs sont débranchés, avant le transport à l'unité des soins intensifs (USI) et, en conséquence, ces épisodes ischémiques passeraient normalement inaperçus. Ce qui suggère un besoin précoce d'analgésie pour diminuer l'intensité de la douleur et le stress associé ou encore, la nécessité de maintenir l'analgésie épidurale une fois le patient libéré de l'USI.

Les auteurs ont aussi recherché les événements spécifiques de la période périopératoire qui pouvaient accompagner l'ischémie myocardique. L'observation la plus importante et aussi la plus inquiétante qui émane de cette étude concerne les périodes à risque particulièrement élevé. Ces périodes comprennent les transferts à I'USI et de l'USI (on pouvait s'y attendre) mais aussi la physiothérapie et la toilette au lit! Comme le font remarquer avec pertinence les auteurs, ces manoeuvres semblent faire plus de mal que de bien! On essaye de détecter l'ischémie cardiaque pour mieux la prévenir. Alors comment peut-on la prévenir à la période postopératoire? Comme les épisodes d'ischémie sont surtout silencieux,' les patients eux-mêmes ne peuvent évidemment en faire état. De plus, il est reconnu que sa détection sur l'ECG usuel par le personnel médical est inefficace. Il est possible que le seul moyen à notre disposition soit constitué par une reconnaissance des périodes de risque élevé prévues ou imprévues comme les auteurs le mentionnent.
Pourquoi ces résultats ne concordent-ils pas avec ceux des études antérieures qui montraient une diminution de la morbidité et de la mortalité chez les patients à qui on avait administré une technique épidurale pour l'anesthésie? Beaucoup de ces études ne se limitaient pas à un seul type de chirurgie comme c'est actuellement le cas. Dans leur étude référence de 1987, Yeager et $a l .^{6}$ ont trouvé que la mortalité postopératoire, les complications cardiovasculaires et respiratoires et les infections graves diminuaient chez les patients sous anesthésie épidurale avec analgésie épidurale postopératoire comparativement aux patients opérés sous anesthésie générale avec analgésie postopératoire à la morphine prn. Cependant, quand on considère la méthode de gestion des patients dans cette étude, on constate que plusieurs facteurs peuvent avoir contribué par mégarde aux résultats et aussi que les études subséquentes ont été dans l'impossibilité de les confirmer.

D'abord, ces auteurs ont étudié un petit nombre de patiens opérés pour une grande variété d'interventions. De plus la technique anesthésique et l'usage du monitorage effractif ont été mal contrôlés au cours de la période périopératoire. En effet, on a déjà démontré que l'utilisation d'un monitorage effractif chez les patients à haut risque pouvait réduire considérablement les complications cardiaques. ${ }^{7}$ Finalement, les protocoles de contrôle de la douleur postopératoire ne pouvaient être comparés. Les patients du groupe épidural étaient suivis quotidiennement par le service de traitement de la douleur aiguë et par contre recevaient des morphiniques prn administrés par le personnel infirmier. En réalité, les soins postopératoires différents peuvent avoir modifé leurs constatations. Des études subséquentes ont invariablement mis l'accent sur l'importance de la gestion postopératoire de la douleur comme facteur de morbidité et de mortalité postopératoires ${ }^{8.9}$ Baron et al. dans leur étude portant sur 173 patients programmés pour une reconstruction chirurgicale aortique ont conclu que l'anesthésie épidurale thoracique combinée à une anesthésie générale légère ne présentaient pas d'avantages sur l'anesthésie générale chez des patients à haut risque. Cependant, pour cette étude, le protocole postopératoire pour l'analgésie était mal intégré au protocole d'étude. En conséquence, trois techniques d'analgésie différentes étaient distribuées également aux deux groupes d'anesthésie épidurale et générale. Les auteurs suggèrent que c'est l'analgésie épidurale postopératoire plutôt que l'anesthésie épidurale peropératoire qui a surtout influencé l'incidence des complication cardiaques postopératoires.

Ils semble bien maintenant que la gestion de la douleur postopératoire en soi ait plus d'influence sur le déroulement de l'évolution postopératoire que le type 
d'anesthésie utilisé pour l'intervention en autant qu'une bonne stabilité hémodynamique est maintenue. Avec un contrôle efficace des variables hémodynamiques, on a démontré en chirurgie cardiaque que la technique anesthésique n'avait pas d'influence sur l'évolution postopératoire. ${ }^{10}$ Faut-il se surprendre si les résultats sont les mêmes lorsqu'ils s'agit de patients cardiaques et de chirurgie non cardiaque?

Quelles autres leçons pouvons-nous tirer de l'article de Garnett et al? D'abord, l'anesthésie régionale ne semble pas fournir de meilleurs résultats qu'une bonne anesthésie générale. Même si elle ne réduit ni la morbidité ni la mortalité cardiaques, de toute évidence, l'anesthésie régionale procure aussi des avantages dont il faut tenir compte, tout en reconnaissant la liberté de choix du patient. Christopherson et al. " ont trouvé que chez les patients soumis à une chirurgie de reconstruction vasculaire non urgente des extrémités inférieures, la proportion de rejet du greffon était plus élevée sous anesthésie générale que sous régionale. Ils ont conclu qu'en diminuant ou en empêchant la réponse nerveuse, hormonale et métabolique au stress, l'anesthésie régionale ramenait vers la normale les processus d'hypercoagulabilité et de fibrinolyse associés à la réponse au stress. Deuxièmement, il devient évident qu'il est temps d'établir des normes cliniques en ce qui concerne la stabilité hémodynamique peropératoire et la gestion de la douleur postopératoire. Finalement, et ce qui est d'une importance capitale, un bon contrôle de la douleur postopératoire représente un facteur essentiel si on veut réduire la morbidité et la mortalité cardiaques.

Enfin, ou peut laisser de côté pour l'instant l'influence respective de l'anesthésie régionale et l'anesthésie générale. II devient incontestable que la question à débattre n'est plus de savoir si la générale est supérieure ou pas, mais plutôt, quel est, dans son ensemble, la meilleure façon de prendre soin du patient à la période périopératoire. En conséquence, la prise en charge anesthésique ne s'arrête pas une fois la porte de la salle d'opération franchie. C'est pourquoi, il faut accorder encore plus d'attention à la période postopératoire.

\section{References}

I Mangano DT, Browner WS, Hollenberg $M$, London $M J$, Tuban JF, Tateo IM. Association of perioperative myocardial ischemia with cardiac morbidity and mortality in men undergoing non-cardiac surgery. N Engl J Med 1990; 323: 1781-8.

2 Siliciano $D$, Mangano DT. Postoperative myocardial ischemia: mechanisms on therapies. In: Estafanous FG (Ed.). Opioids in Anesthesia II. Stoneham, Mass: Butterworth 1990: 164-78.

3 Herizer NR. Basic data concerning associated coronary disease in peripheral vascular patients. Ann Vasc Surg 1987; 1: 616-20.

4 Hertzer NR, Beven EG, Young JR, et al. Coronary artery disease in peripheral vascular patients. A Classification of 1000 coronary angiograms and results of surgical management. Ann Surg 1984; 199: 223-33.

5 Garnett RL, Barber GG, McPhail NV, Boisvert D. Perioperative ischemia in aortic surgery: combincd epidural general anaesthesia and epidural analgesia vs general anaesthesia and IV analgesia. Can J Anaesth 1996; 43: 769-77.

6 Yeager MP, Glass DD, Neff RK, Brinck-Johnsen T. Epidural anesthesia and analgesia in high-risk surgical patients. Anesthesiology 1987; 66: 729-36.

7 Rao TLK, Jacobs $K H$, EL-Etr AA. Reinfarction following anesthesia in patients with myocardial infarction. Anesthesiology 1983; 59: 499-505.

8 Baron JF, Bertrand $M$, Barré E, et al. Combined epidural and general anesthesia versus general anesthesia for abdominal aortic surgery. Anesthesiology 1991; 75: 611-8.

9 Tuman KJ, McCarthy RJ, March RJ, DeLaria GA, Patel $R V$, Ivankovich $A D$. Effects of epidural anesthesia and analgesia on coagulation and outcome after major vascular surgery. Anesth Analg 1991; 73: 696-704.

10 Slogoff $S$, Keats $A S$. Randomized trial of primary anesthetic agents on outcome of coronary artery bypass operations. Anesthesiology 1989; 70: 179-88.

11 Christopherson R, Beattie C, Frank SM, et al. Perioperative morbidity in patients randomized to epidural or general anesthesia for lower extremity vascular surgery. Anesthesiology 1993; 79: 422-34. 